\title{
Great SAND DUNES NaTional MONUMENT VEgeTATION PATTERNS
}

\author{
E. DURANT MCARTHUR $\bullet$ STEWART C. SANDERSON \\ U. S. D. A. FOREST SERVICE INTERMOUNTAIN RESEARCH STATION \\ SHRUB SCIENCES LABORATORY $\bullet$ PROVO
}

\begin{abstract}
This project is designed to characterize and map the vegetation of the Great Sand Dunes National Monument, Colorado (GSDNM) and to determine if the vegetated areas in the dune field are permanent, temporary, or migratory. It is not known if the vegetation around the dunes is encroaching on the dunes, being replaced by the dunes or is stable. There are also concerns about the possible effect a proposed water export project adjacent to GSDNM would have on the dunes and their vegetation.
\end{abstract}

\section{$\checkmark$ METHODS}

Vegetation patterns were characterized by establishing a grid system of plots throughout GSDNM based on section corners and ongoing sand trap dune movement assays (Fig. 1A). The grid was supplemented by additional plots in smaller and more unique vegetative communities. Fewer plots were put in the large vegetatively depauperate sand dune areas. Circular plots $(0.01 \mathrm{ha})$ were marked with the placement of $30 \mathrm{~cm}$ steel bars. Plot sampling was by the releve methodology developed by the ZurichMontpellier School of Plant Ecology (Shimwell 1971, Harper et al. 1988). Each plot was photographed, its parental geological material recorded (Johnson 1969, Tweto 1979), vascular plant species identified (Welsh et al. 1987, Weber 1990) and classified by cover class $(<1 \%,=+, 1-5 \%=1,6-25 \%=2$, $26-50 \%=3,51-75 \%=4,76-95 \%=5$, > $95 \%=6$; modified from Daubenmire 1959) and sociability class (single, widely spaced individuals $=$ 1 , small groups $=2$, small patches $=3$, extensive patches $=4$, nearly pure stands $=5$; Harper et al. 1988), and a summary of the plot's cover made by percent tree layer, shrub layer, herb layer, cryptogamic layer, litter, rock $(>1 \mathrm{~cm})$, and bare soil.

Plant communities were objectively identified by using multivariate statistical techniques we developed. We used two complementary clustering methods. Both methods are based on the co-occurrence of species. However, in the prevalence method, Prevalence Affinity, $P_{A}=N_{S 2} / N_{S 1}$, where $N_{S 1}=$ number of plots with species 1 in the species plots under consideration and $\mathrm{N}_{\mathrm{S}_{2}}=$ number of plots with species 2 co-occurring with species 1 . In the uniqueness method, corrected for random expectation, Uniqueness Affinity, $\mathrm{U}_{\mathrm{A}}=\left(\mathrm{N}_{\mathrm{S2}} *\right.$ $\left.\mathrm{N}_{\mathrm{TS}}\right) /\left(\mathrm{N}_{\mathrm{S1}} * \mathrm{~N}_{\mathrm{T} 1}\right)$, where $\mathrm{N}_{\mathrm{TS}}=$ total number of plots in the study, $\mathrm{N}_{\mathrm{T} 1}=$ total number of plots of species 1 in the complete study, and the other symbols are as in the $\mathrm{P}_{\mathrm{A}}$ model.

Concurrent with our travel to and from study plots we supplemented the existing list of vascular plants (Great Sand Dunes National Monument Staff 1986) by collecting herbarium specimens of plants heretofore unknown from GSDNM which have been deposited in the herbaria of GSDNM, the Shrub Sciences Laboratory (SSLP), and Snow College. 


\section{$\checkmark \quad$ RESULTS AND DISCUSSION}

Data from the 118 plots (Fig. 1A) subjected to $P_{A}$ and $\mathrm{U}_{\mathrm{A}}$ clustering sorted the plots into three prevalance clusters (Stabilized and Active Dunes, Wetlands, and Mountainside) and six complementary uniqueness clusters, two each for the prevalance clusters (Active Dunes, Stabilized Dunes, Marsh, Streambank, and two Mountainside clusters) (Table 1). The two clustering proceedures objectively classify natural vegetation communities (Fig. 1B, 1C, 1D). The dry sand communities are either on the large active dune mass or on adjacent smaller stabilized dunes. All the active dune species are found on the stabilized dunes as well but the converse is not true. The wetland communities are along the steam courses or at other locations of surface water or high water tables. The tightest clustering in the whole study is the Marsh Uniqueness cluster. Mountainside communities are poorly differentiated because the relative small area included few plots for our study. Mountainside communities are on the slopes of the Sangre de Cristo Mountains which flank GSDNM on the north and east and are included only at the edge of GSDNM. The usefulness of our clustering method is illustrated by comparing means within cluster vs. means between clusters: $P_{A}$ is 217.8 vs. $78.2 ; U_{A}$ is 291.2 vs. 93.1. We discovered 59 species from 26 familes new to the flora of the monument.

\section{- ADDITIONAL RESEARCH}

The plot data are "ground truth" data. We will match these ground truth data points with vegetative patterns characteristic on aerial photographs taken in 1936 and 1990. We can then make an assessment of stability of the dunes and vegetative patterns and produce vegetation maps.

The 59 species we added to the known flora of GSDNM represent an increase of $20 \%$ over the number of species previously known to occur at GSDNM (Great Sand Dunes National Monument Staff 1986). This increased number was obtained in an incidental way as we recorded plot data and traveled to and from plots. We believe a systematic search for new species would be productive.

\section{$\checkmark$ ACKNOWLEDGEMENTS}

Personnel at GSDNM were gracious and helpful in every way; we especially thank Bill Haviland, Fred Bunch, and Bill Wellman for their assistance. Clyde Blauer, Jeff Ott, Gary Jorgensen, Steve Briggs, and Craig Ogden capably assisted with data collection and summarization.

\section{$\checkmark$ Literature Cited}

Daubenmire, R. 1959. A canopy-coverage method of vegetational analysis. Northwest Sci. 33:43-64.

Great Sand Dunes National Monument Staff. 1986. List of vascular plants. Typescript manuscript, Great Sand Dunes National Monument, Colorado.

Harper, K. T., S. C. Sanderson, and E. D. McArthur. 1988. Vegetation communities of Zion National Park. Pages 191-196 In: University of Wyoming National Park Service Research Center Eleventh Annual Report, 1987. Laramie.

Johnson, R. B. 1969. Geologic map of the Trinidad Quadrangle, south-central Colorado. U. S. Geological Survey Miscellaneous Geological Inventory Map I-558.

Shimwell, D. W. 1971. The description and classification of vegetation. University of Washington Press, Seattle.

Tweto, O. 1979. Geologic map of Colorado. U. S. Geological Survey, Reston, Virginia.

Welsh, S. L., N. D. Atwood, S. Goodrich, and L. C. Higgins (eds.). 1987. A Utah flora. Great Basin Naturalist Memoirs 9:1-894.

Weber, W. A. 1990. Colorado flora: eastern slope. University Press of Colorado, Niwot. 
Table 1. Vegetative communities as determined by prevalence and uniqueness clustering.

Prevalence Clustering

Stabilized and Active Dune Species (78 plots, plus 8 mixed)

\begin{tabular}{lcc}
\hline Clustered species & Plots & Relative affinity \\
\cline { 2 - 3 } Redfieldia flexuosa & 61 & 207.5 \\
Psoralea lanceolata & 60 & 181.5 \\
Oryzopsis hymenoides & 56 & 199.5 \\
Helianthus petiolaris & 56 & 217.0 \\
Chrysothamnus nauseosus & 49 & 176.6 \\
Senecio sparteoides & 47 & 160.6 \\
Lygodesmia juncea & 37 & 211.1 \\
Sporobolus cryptandrus & 31 & 197.8 \\
Stipa comata & 30 & 158.6 \\
Corispermum nitidum & 28 & 240.7 \\
Oenothera coronopifolia & 28 & 222.2 \\
Cryptantha fedleri & 18 & 174.6 \\
Yucca glauca & 16 & 165.1 \\
Ambrosia acanthicarpa & 15 & 226.1 \\
Muhlenbergia pungens & 14 & 208.1 \\
Crypthantha jamesii & 13 & 201.8 \\
Salsola kali & 13 & 196.8 \\
Penstemon angustifolius & 11 & 198.9 \\
Eriogonum cermuum & 10 & 201.2 \\
Lithospermun incisum & 9 & 200.1 \\
<6 plots (23 species & - & 225.0 \\
Weakly Clusterd (9 sp.) & - & 129.0 \\
Shared: -none- & - & -
\end{tabular}

Wetlands (13 plots, plus 11 mixed)

\begin{tabular}{lcc}
\hline Clustered species & Plots & Relative affinity \\
\cline { 2 - 3 } Agropyron smithii & 14 & 176.8 \\
Juncus balticus & 11 & 294.7 \\
Populus angustifolia & 10 & 217.2 \\
Bromus anomalus & 10 & 197.0 \\
Poa praetensis & 10 & 265.1 \\
Taraxacum officinale & 10 & 259.6 \\
Ribes leptanthum & 9 & 217.0 \\
Rosa woodsii & 9 & 284.9 \\
Salix exigua & 8 & 294.0 \\
Rhus trilobata & 7 & 177.3 \\
Carex sp. & 7 & 278.7 \\
Achillea lanulosa & 7 & 285.0 \\
Populus tremuloides & 6 & 260.8 \\
Alnus tenuifolia & 6 & 317.0 \\
Agropyron trachycaulum & 6 & 264.4 \\
Agrositis stolonifera & 6 & 315.6 \\
Muhlenbergia asperifolia & 6 & 284.7 \\
Aster chilensis & 6 & 259.5 \\
Epilobium glandulosum & 6 & 317.0 \\
Equisetum arvense & 6 & 317.0 \\
Lactuca tatarica & 6 & 249.3 \\
Smilacina stellata & 6 & 227.1 \\
<6 plots (94 species) & - & 295.1 \\
Weakly Clustered & - & 126.3 \\
Shared:Mountainside (3 sp.) & - & 157.3 \\
& - &
\end{tabular}

Uniqueness Clustering

Active Dunes (29 plots, plus 47 shared with Stabilzed Dunes, plus

9 mixed)

\begin{tabular}{lll}
\hline Clusterd species & Plots & Relative affinity
\end{tabular}

Redfieldia flexuosa $\quad 61 \quad 253.2$

Helianthus petiolaris $\quad 56 \quad 248.5$

$\begin{array}{lll}\text { Lygodesmia juncea } & 37 & 259.8\end{array}$

$\begin{array}{lll}\text { Corispermum nitidum } & 28 & 362.5\end{array}$

$\begin{array}{lll}\text { Oenothera coronopifolia } & 28 & 282.0\end{array}$

Ambrosia acanthicarpa $\quad 15 \quad 379.1$

Salsola kali $\quad 13 \quad 278.6$

<6 plots (4 species) _ _ $\quad$ - $\quad 375.3$

Weakly Clustered (5 sp.) _ _ $\quad 195.8$

Shared:Stabilized Dunes (11 sp.)-- $\quad 258.1$

Stabilized Dunes (47 plots, all shared with Active Dunes.

\begin{tabular}{lcc}
\hline Clustered species & Plots & $\begin{array}{r}\text { plus 7 } \\
\text { Relative a }\end{array}$ \\
\cline { 2 - 3 } & & \\
Sporobolus cryptandrus & 31 & 249.7 \\
Muhlenbergia pungens & 14 & 257.2 \\
Cryptantha jamesii & 13 & 266.5 \\
Eriogonum cernuum & 10 & 244.7 \\
Lithospermum incisum & 9 & 289.2 \\
<6 plots (8 species) & - & 265.9 \\
Weakly Clustered (9 sp.) & - & 200.7 \\
Shared:Active Dunes (11 sp.) & - & 265.6
\end{tabular}

Marsh (2 plots, plus 4 shared with Streambands, plus 8 mixed)

\begin{tabular}{lcc}
\hline Clustered species & Plots & Relative affinity \\
\hline & & \\
Agropyron smithii & 14 & 272.8 \\
Muhlenbergia asperifolia & 6 & 569.8 \\
Lactuca tatarica & 6 & 396.4 \\
<6 plots (22 species) & - & 608.2 \\
Weakly Clustered *2 sp.) & - & 194.4 \\
Shared:Streambank (48 sp.) & - & 310.8
\end{tabular}

Streambank (6 plots, plus 4 shared with Marshes, plus 10 mixed)

\begin{tabular}{lcc}
\hline Clustered species & Plots & Relative affinity \\
\cline { 2 - 3 } Populus angustifolius & 10 & 251.9 \\
Poa praetensis & 10 & 289.9 \\
Ribes leptanthum & 9 & 239.2 \\
Rosa woodsii & 9 & 326.5 \\
Salix exigua & 8 & 303.8 \\
Carex sp. & 7 & 271.6 \\
Populus tremuloides & 6 & 322.0 \\
Alnus tenuifolia & 6 & 367.5 \\
Agropyron trachycaulum & 6 & 289.4 \\
Equisetum arvense & 6 & 367.5 \\
Smilacina stellata & 6 & 261.7 \\
<6 plots (26 species) & -- & 339.3 \\
Weakly Clustered (6 sp.) & - & 195.1 \\
Shared:Marsh (48 sp.) & -- & 292.7
\end{tabular}


Table 1. (cont)

Mountainside (15 plots, plus 11 mixed)

\begin{tabular}{|c|c|c|}
\hline Clustered specied & Plots & Relative affinity \\
\hline Erysimum asperum & 28 & 181.7 \\
\hline Opuntia polycantha & 25 & 171.9 \\
\hline Symphoricarpos oreophilus & 22 & 182.3 \\
\hline Artemisia frigida & 22 & 180.5 \\
\hline Heterotheca villosa & 20 & 180.3 \\
\hline Juniperus scopulorum & 19 & 170.1 \\
\hline Bouteloua gracilis & 19 & 164.1 \\
\hline Senecio tridenticulatus & 19 & 159.8 \\
\hline Sitanion hystrix & 15 & 190.0 \\
\hline Pinus edulis & 13 & 203.4 \\
\hline Carex rossii & 13 & 212.9 \\
\hline Festuca ovina & 13 & 201.1 \\
\hline Machaeranthera canescens & 13 & 183.5 \\
\hline Poa fendleriana & 12 & 201.5 \\
\hline Eriogonum jamesii & 12 & 217.6 \\
\hline Muhlenbergia montana & 11 & 221.5 \\
\hline Androsace septentrionalis & 11 & 202.2 \\
\hline Cercocarpus montanus & 10 & 223.6 \\
\hline Hododiscus dumosus & 10 & 216.2 \\
\hline Ribes cereum & 10 & 213.1 \\
\hline Oryzopsis micrantha & 10 & 184.1 \\
\hline Chenopodium fremontii & 10 & 176.1 \\
\hline Senecio fendleri & 10 & 168.5 \\
\hline Pseudotsuga menziesii & 9 & 202.5 \\
\hline Descurania pinnata & 9 & 202.3 \\
\hline Gilia aggregata & 9 & 170.8 \\
\hline Abies concolor & 8 & 193.7 \\
\hline Arabis lignifera & 8 & 217.9 \\
\hline Arabis hoelboellii & $\cdot 7$ & 214.5 \\
\hline Chrysothamnus viscidiflorus & 6 & 181.3 \\
\hline Ribes aureum & 6 & 209.7 \\
\hline Koeleria nitida & 6 & 226.7 \\
\hline Gilia pinnatifida & 6 & 203.1 \\
\hline Lappula redowskii & 6 & 185.7 \\
\hline Penstemon barbatus & 6 & 206.1 \\
\hline$<6$ plots (51 species) & - & 211.7 \\
\hline Weakly Clustered (9 sp.) & - & 131.4 \\
\hline Shared:Wetlands (3 sp.) & - & 160.7 \\
\hline
\end{tabular}

Mountainside A (0 plots, 15 shared with B, plus 11 mixed)

\begin{tabular}{lcc}
\hline Clustered species & Plots & Relative af \\
\cline { 2 - 3 } Poa fendleriana & 12 & 245.2 \\
Androsace septentrionalis & 12 & 226.0 \\
Ribes cereum & 10 & 242.4 \\
Abies concolor & 8 & 230.2 \\
Penstemon barbatus & 6 & 262.4 \\
<6 plots (5 species) & - & 252.3 \\
Weakly Clustered (25 sp.) & - & 190.3 \\
Shared:Mountainside B (22 sp.) & - & 261.2
\end{tabular}

Mountainside B ( 0 plots, 15 shared with A, plus 11 mixed)

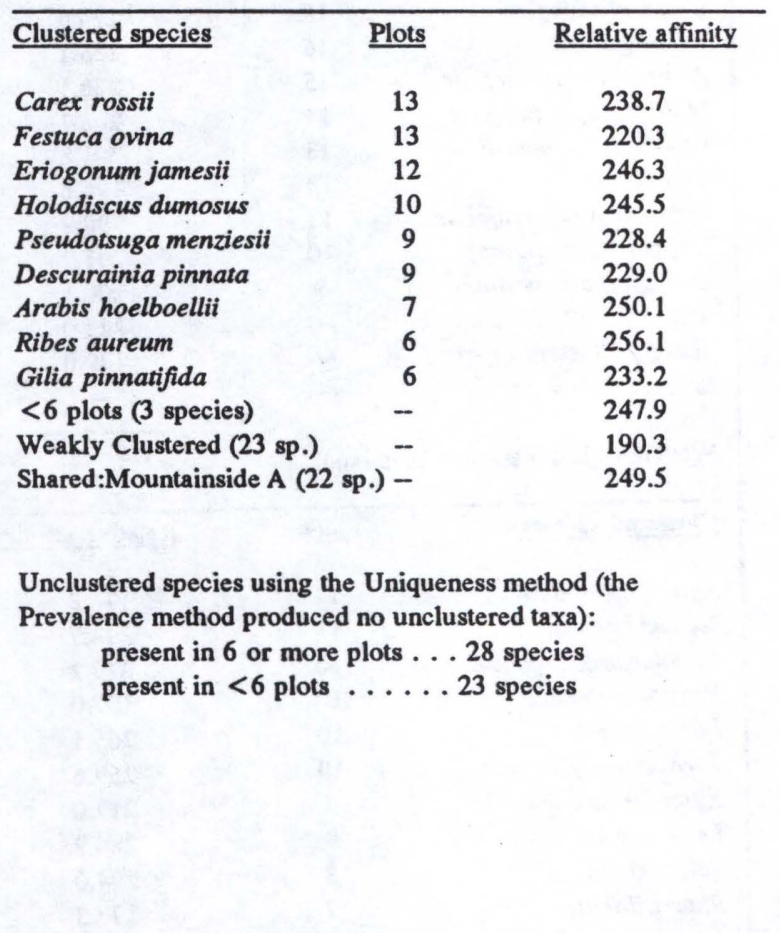




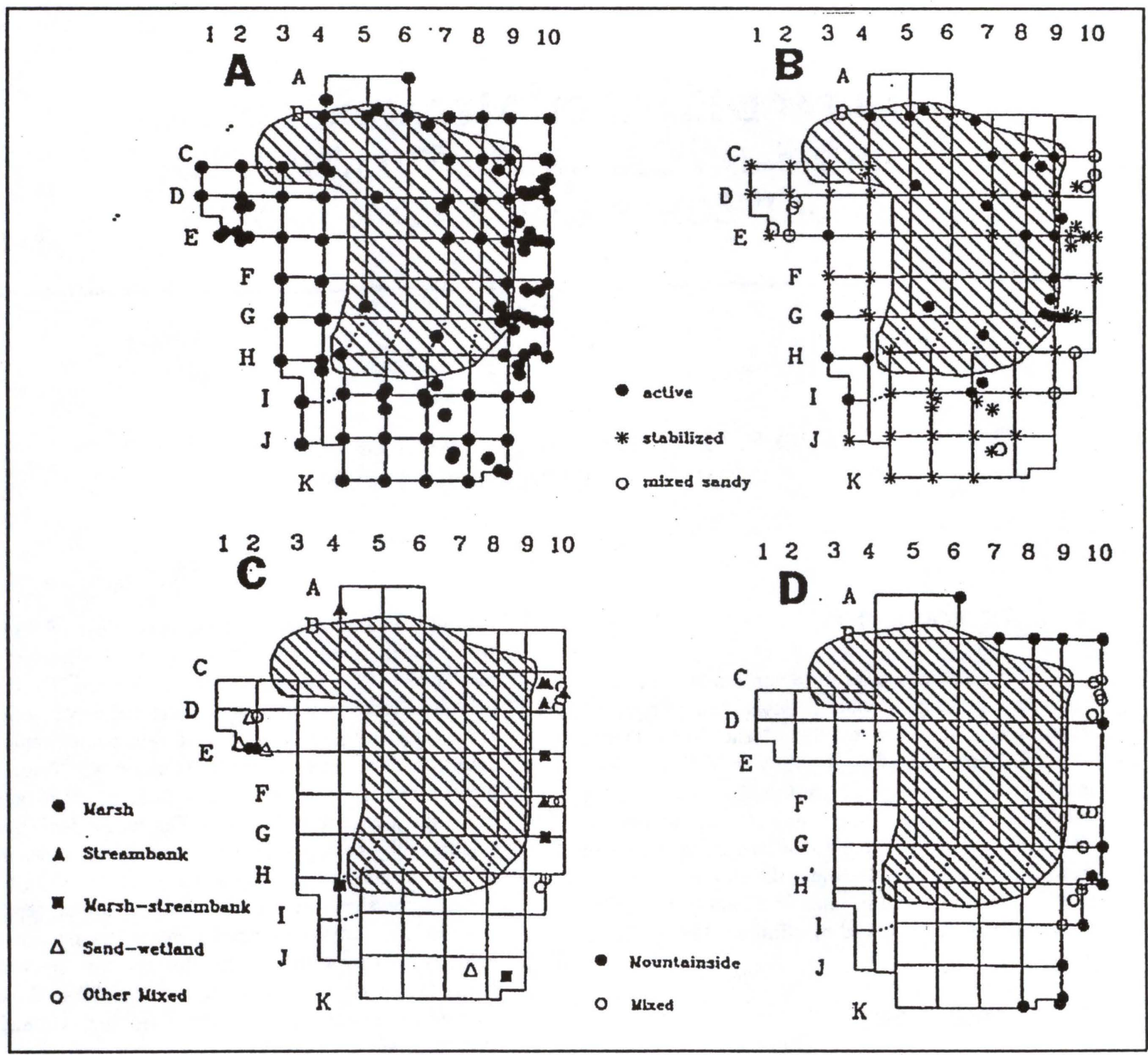

Figure 1. Locations of study plots and vegetation clusters, Great Sand Dunes National Monument. 1A. Location of vegetation survey plots. The hatched area outlines the main dune mass. The monument boundry follows the outside line. 1B. Location of dunes vegetation cluster. 1C. Location of wetlands vegetation cluster. 1D. Location of mountainside vegetation cluster. 